Clare Elizabeth Tange*, Bridget Johnson-Brett, Alex Cook, Patrick Stordeur, Fabian Brohet, Stephen Jolles, Rachel Steven, Mark Ponsford, Andrew Roberts, Tariq El-Shanawany, Stephen Harding, Gregg Wallis and Antony Richard Parker

\title{
Quantification of human complement C2 protein using an automated turbidimetric immunoassay
}

https://doi.org/10.1515/cclm-2017-1068

Received November 17, 2017; accepted March 9, 2018; previously published online May 5, 2018

\section{Abstract}

Background: The measurement of complement components is clinically useful where a deficiency is suspected, or where excessive activation and consumption are present in disease. C2 deficiency carries an increased risk of developing systemic lupus erythematosus, recurrent infections and atherosclerosis. In this study, we have evaluated The Binding Site's Human Complement C2 SPAPLUS ${ }^{\circledR}$ assay.

Methods: Linearity was tested using 13 sample dilutions covering the standard measuring range. Within- and between-assay variabilities were calculated using five samples with different $\mathrm{C} 2$ concentrations. The correlation between $\mathrm{C} 2$ concentrations in EDTA-plasma and serum was assessed, as was the correlation between C2 measurements by the automated assay and radial immunodiffusion. C2 concentrations were compared with $\mathrm{CH} 50$ activity, and quantified in individuals with homozygous or heterozygous C2 deficiency, acquired angioedema and patients with chronic inflammatory conditions.

Results: The assay was linear across the measuring range $(3.8-42.3 \mathrm{mg} / \mathrm{L})$. Intra- and interassay variability were $2.3 \%-3.8 \%$ and $0 \%-3.3 \%$, respectively. Comparison between $\mathrm{C} 2$ measurements in EDTA-plasma and serum provided a strong correlation $\left(\mathrm{p}<0.0001, \mathrm{R}^{2}=0.82\right.$, slope 0.92 ), as did the correlation between the automated and radial immunodiffusion methods $\left(\mathrm{p}<0.0001, \mathrm{R}^{2}=0.89\right.$,

\footnotetext{
*Corresponding author: Clare Elizabeth Tange, The Binding Site Group Limited, 8 Calthorpe Road, B15 1QT, Birmingham, UK, E-mail: clare.tange@bindingsite.co.uk Bridget Johnson-Brett, Alex Cook, Stephen Harding, Gregg Wallis and Antony Richard Parker: The Binding Site Group Limited, Birmingham, UK

Patrick Stordeur and Fabian Brohet: Immunobiology Clinic, Erasme Hospital, Université Libre de Bruxelles, Brussels, Belgium Stephen Jolles, Rachel Steven, Mark Ponsford, Andrew Roberts and Tariq El-Shanawany: Immunodeficiency Centre for Wales, University Hospital of Wales, Cardiff, UK
}

slope 1.07). A positive correlation between C2 concentration and CH50 activity was demonstrated $(\mathrm{p}<0.0001$, $\left.\mathrm{R}^{2}=0.48\right)$. Significant differences were observed between the median C2 concentrations obtained in healthy controls and the patient clinical samples, with homozygous C2-deficient patients giving below detectable results.

Conclusions: This C2 SPAPLUS ${ }^{\circledR}$ assay allows the automated, rapid and precice quantification of complement $\mathrm{C} 2$ protein and could therefore be considered as a replacement for older, more time-consuming methods.

Keywords: acquired angioedema (AAE); C2; complement; complement deficiency; hereditary angioedema (HAE); SPAPLUS; systemic lupus erythematosus (SLE); turbidimetry.

\section{Introduction}

The complement cascade is a crucial part of the innate immune system and plays a key role in host defense against pathogenic infections. There are three convergent complement pathways [classical, alternative and mannosebinding lectin (MBL)] that consist of more than 30 soluble and membrane-bound proteins $[1,2]$. These pathways are tightly regulated activation cascades that result in the production of effector molecules with diverse biological functions. Complement activation triggers inflammation by recruiting and activating immune cells, promotes the phagocytosis and clearance of pathogens through opsonization and can result in lysis of pathogen cells through the formation of a membrane attack complex [3, 4]. The complement system is also involved in the clearance of apoptotic cells and immune complexes and in amplifying adaptive immune responses $[1,2,5]$.

$\mathrm{C} 2$ is one of the key proteins in the classical and MBL pathways of complement activation. C2 binds to membrane bound C4b and is subsequently cleaved by $\mathrm{C} 1 \mathrm{~s}$ or MASP2 $[6,7]$, generating $\mathrm{C} 2 \mathrm{~b}$, which is released into the fluid phase, and C4bC2a (a C3-convertase). This is a crucial and central step in complement activation. Each C3 convertase cleaves multiple C3 proteins, resulting in 
the production of important effector molecules - C3a and C3b [2].

The measurement of individual complement proteins is clinically important where a deficiency is suspected, or where excessive activation and consumption are present in disease [8-10]. Homozygous C2 deficiency is the most common inherited complement deficiency, with an estimated prevalence of 1 in 10-20,000 in populations of European descent [11, 12]. The most frequent C2 deficiency is caused by a 28-base pair (bp) deletion in the $\mathrm{C} 2$ gene, which results in a complete lack of $\mathrm{C} 2$ protein synthesis in homozygous cases [13]. Although many C2-deficient individuals appear entirely healthy [12], homozygous C2 deficiency is associated with an increased risk of developing systemic lupus erythematosus (SLE) [14, 15], atherosclerosis [10] and an increased susceptibility to infections caused by encapsulated bacteria such as Streptococcus pneumoniae, Neisseria meningitidis or Haemophilus influenzae [16-18].

The measurement of $\mathrm{C} 2$ protein concentration is a useful tool in aiding the diagnosis of C2 deficiency and a key first step in the evaluation of a patient found to have reduced or absent classical pathway function. Current methods used include electroimmunoassay (EIA) [19] and radial immunodiffusion (RID) [20, 21]. These assays have proven valuable for the measurement of $\mathrm{C} 2$, but there is the need for a more automated method. Here we describe the characteristics of an automated assay for the measurement of $\mathrm{C} 2$ protein concentration on the SPAPLUS ${ }^{\circledR}$ turbidimetric analyzer.

\section{Materials and methods}

\section{Plasma and serum samples}

Plasma samples were collected by venepuncture into EDTA blood collection tubes. For serum samples, blood was collected by venepuncture and allowed to clot naturally, after which the serum was separated to prevent hemolysis. Where possible, samples were tested immediately after collection; otherwise (unless specifically stated), they were stored at -20 immediately and subjected to one freeze/thaw cycle.

Serum and plasma samples were obtained from several sources for these studies.

Analytical study: Samples from healthy adult blood donors (age range 19-81 years) were purchased from Quest Biomedical (Solihull, UK). Sample collection was approved by the Institution Ethics Review Board (\#05142), with all donors providing written informed consent. These samples were used for assay validation experiments and development of the normal adult reference range. Where required, pooled samples (from three to five donors) were used to generate samples of various $\mathrm{C} 2$ concentrations.
Matrix comparison: Residual and anonymous paired EDTA-plasma and serum samples from patients referred for immunological investigation ( $n=119$, age range $0.7-85$ years) were obtained from the Immunobiology Clinic of the Erasme Hospital, Belgium. Sample collection and use was approved by the Ethics Committee of the Erasme Hospital, Belgium. Samples were used for the matrix comparison and the comparison of $\mathrm{C} 2$ protein concentration to $\mathrm{CH} 50$ functional activity.

Paired EDTA-Plasma and serum samples were collected from consenting healthy adult blood donors at The Binding Site $(n=20$, age range $25-57$ years). These were used to examine the stability of $\mathrm{C} 2$ protein measurements in the two matrices over 3 days.

C2 assay comparison: Serum samples were collected from consenting healthy adult blood donors at The Binding Site $(n=24$, age range 27-47 years).

Anonymous stored serum samples from patients with confirmed or suspected $\mathrm{C} 2$ deficiency ( $\mathrm{n}=7$, age range 5-64 years) were obtained from the Immunodeficiency Centre for Wales.

Both sets of samples were run on the automated C2 assay and RID assay.

Clinical study: Serum samples were collected from consenting healthy adult blood donors at The Binding Site $(n=20$, age range 25-55 years).

Additional residual and anonymous clinical samples were obtained from the Clinical Immunology Service, University of Birmingham ( $n=32$, age range $28-83$ years). Collection of residual samples and de-identified patient data was approved by the Institution Ethics Review Board (REC Reference: RRK4136 and UHB R\&D Project Reference 2002/201 "Blood donations for use in clinical assays").

Anonymous stored serum samples from patients with previously diagnosed homozygous ( $n=4$, age range 5-64 years) or heterozygous C2 deficiency ( $n=1,20$ years) or acquired angioedema (AAE, $n=2$, age range $65-78$ years) were obtained from the Immunodeficiency Centre for Wales.

\section{Production of $\mathrm{C} 2$ antiserum}

C2 isolation from human plasma: C2 was purified from pooled fresh-frozen plasma. Pooled plasma was dialyzed against calcium chloride to precipitate the $\mathrm{C} 1$ complex, fractionated by sequential ammonium sulfate precipitation and ion exchange chromatography (SP-Sepharose and Q-Sepharose, GE Healthcare, Chicago, IL, USA), and then further purified by size exclusion chromatography (Superdex S200, GE Healthcare). Final purity was assessed by SDS-PAGE, followed by silver staining or immunoblotting. SDS-PAGE was conducted using 4\%-12\% NUPAGE ${ }^{\circledR}$ gels (Thermofisher Scientific Inc., MA, USA) with a pH 6.0 MOPS running buffer as described in the manufacturer's instructions. Silver staining of the gels was accomplished using the Bio-Rad silver stain kit (Bio-Rad, Watford, UK). Purified C2 protein was quantified by N-terminal amino acid compositional analysis (Alta Bioscience, Birmingham, UK).

Conjugated C2 antiserum preparation: Sheep anti-human C2 polyclonal antiserum was generated by subcutaneous immunization with $150 \mu \mathrm{g}$ of human C2 antigen mixed with complete Freund's adjuvant, and subsequent boosting at monthly intervals with $30 \mu \mathrm{g}$ antigen in incomplete Freund's adjuvant. The IgG fraction was isolated 
from sheep antiserum by caprylic acid precipitation and was affinity purified to specificity using a C2 affinity column (CNBr-Sepharose). $\mathrm{F}(\mathrm{ab})_{2}$, fragments were prepared using pepsin and were conjugated to $200 \mathrm{~nm}$ carboxyl-modified polystyrene particles with 1-ethyl-3-(3dimethylaminopropyl) carbodiimide hydrochloride (Thermofisher).

\section{Analytical techniques}

C2 concentrations were measured using the automated Human Complement C2 SPAPLUS ${ }^{\circledR}$ assay. CH50 classical pathway activity was measured using the CH50 SPAPLUS ${ }^{\circledR}$ assay. Both of these assays are automated turbidimetric assays and were run on the SPAPLUS ${ }^{\circledast}$ turbidimeter. For the $\mathrm{C} 2$ assay comparison, $\mathrm{C} 2$ concentrations were measured using the automated Human Complement C2 SPAPLUS ${ }^{\circledR}$ assay and the Human Complement C2 NL BINDARID ${ }^{\mathrm{TM}}$ RID Kit. All assays were performed following the manufacturer's instructions (The Binding Site Ltd., Birmingham, UK).

Assay validation: Assay validation procedures were adapted from the Clinical and Laboratory Standards Institute guidelines. Pooled EDTA-plasma samples (usually 3-5 donors/pool) from healthy adult blood donors were used. For the assessment of linearity, a high EDTAplasma sample pool $(54.8 \mathrm{mg} / \mathrm{L})$ was diluted to 13 consecutive lower analyte concentrations using a second EDTA-plasma sample pool $(0.9 \mathrm{mg} / \mathrm{L})$ to cover the measuring range of the assay. Imprecision analysis was carried out with five independent samples (7.7, 7.9, 11.5, 21.2 and $35.2 \mathrm{mg} / \mathrm{L}$ ). Low-level samples were generated by mixing EDTA-plasma with a complement depleted sample pool (heat inactivated for $2 \mathrm{~h}$ at $60^{\circ} \mathrm{C}$ ).

Percentage coefficient of variation (\% CV) and standard deviations (SDs) for within-run precision were calculated from 20 individual measurements for each sample in a single run. For the other precision calculations, each sample was assayed in duplicate, with two runs per day over 21 days, giving a total of 84 readings per sample. Between-batch variability for three different batches, between instrument variability across three different instruments, betweenrun, between-day and total precision were calculated from the 84 measurements.

Interference analysis was performed by spiking hemoglobin (5 g/L), bilirubin (200 mg/L), chyle (1500 FTU), triglyceride $(1000 \mathrm{mg} / \mathrm{dL})$ or intralipid $(500 \mathrm{mg} / \mathrm{dL})$ into EDTA-plasma samples with three different $\mathrm{C} 2$ concentrations (for hemoglobin, bilirubin and chyle: $7.8,10.4$ and $27.8 \mathrm{mg} / \mathrm{L}$; for intralipid and triglyceride; 7.5, 10.5 and $25.1 \mathrm{mg} / \mathrm{L} \mathrm{C2}$ ). Controls were generated by spiking samples with equivalent volumes of saline. The assay was deemed to have passed the interference assessment if the $\mathrm{C} 2$ concentration after addition of the potential interfering substances was $< \pm 10 \%$ of the original value in the control sample.

Normal reference ranges: The reference range was established by measuring the $\mathrm{C} 2$ concentrations in EDTA-plasma samples taken from 120 healthy adult blood donors as mentioned above. These results were used to calculate the central 95th percentile range and the skewness and kurtosis of the distribution.

Matrix comparison: Paired EDTA-plasma and serum samples were analyzed using the turbidimetric C2 assay and compared using Bland-Altman and Passing-Bablok analyses. To assess the stability of $\mathrm{C} 2$ measurements in the two matrices, the 20 paired samples from healthy volunteers were run on the C2 SPAPLUS ${ }^{\circledR}$ assay on the day of collection, and after $1-3$ days storage at $4^{\circ} \mathrm{C}$. Concentrations between the matrices were evaluated using Passing-Bablok.

Comparison to CH50 activity: Serum samples were tested for $\mathrm{CH} 50$ complement activity and compared to $\mathrm{C} 2$ protein concentration using Bland-Altman and Passing-Bablok analyses.

Clinical study: Serum samples from healthy controls $(n=20)$, patients attending the Clinical Immunology Department, Birmingham QE Hospital $(n=32)$, and patients with C2 deficiency $(n=5)$ or acquired angioedema $(n=2)$ were assayed using the $\mathrm{C} 2$ turbidimetric assay described above. The patients attending Birmingham QE hospital suffered from a range of conditions including SLE, coeliac disease and Sjögren's syndrome.

\section{Statistical analysis}

All graphs and statistical analysis were generated using Graph Pad Prism statistical software version 5.04. or Analyse-it ${ }^{\circledR}$ for Microsoft Excel. The linearity, reference intervals and assay validation calculations (\% CVs and SD) were calculated using Analyse-it. The matrix comparison and $\mathrm{C} 2 / \mathrm{CH} 50$ results were analyzed using Bland-Altman and/or Passing-Bablok analyses (Analyse-it). Differences in the C2 concentrations between disease groups and healthy controls were assessed using Kruskal-Wallis with Dunn's Multiple Comparison Test (Graphpad Prism). A p $<0.05$ was considered statistically significant.

\section{Results}

\section{Development of antisera}

Purity of the C2 antigen was shown by a single band of $105 \mathrm{kDa}$ on silver stained SDS-PAGE gels and was deemed to have $>95 \%$ purity (Figure 1A). N-terminal sequencing

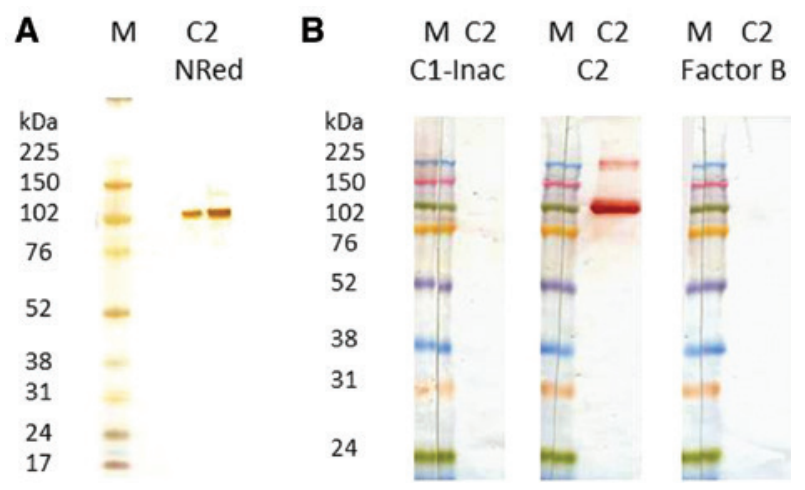

Figure 1: Purity of $\mathrm{C} 2$ antigen.

Marker (M) or C2 protein (C2) were added to gels and the purity of $\mathrm{C} 2$ antigen assessed by (A) non-reducing (Nred) SDS-PAGE at concentrations of 200 and $500 \mathrm{ng}$ per lane. Silver staining was used for detection. (B) $500 \mathrm{ng}$ was analyzed by immunoblotting with the antisera indicated (C1 inactivator, $\mathrm{C} 2$, or Factor $\mathrm{B}$ ). 
confirmed C2 identity. The resultant antiserum recognized the $\mathrm{C} 2$ protein band upon western blotting of human plasma. No major contaminating proteins were detected in the purified C2 preparation (results shown for Factor $\mathrm{B}$ and $\mathrm{C} 1$ inactivator, Figure 1B). No bands were detected with C2-depleted serum (data not shown).

\section{Assay validation}

The automated C2 assay was linear between 2.3 and $54.8 \mathrm{mg} / \mathrm{L}\left(\mathrm{y}=1.0 \times-0.01, \mathrm{R}^{2}=0.99\right)$, covering the measuring range of $3.8-42.3 \mathrm{mg} / \mathrm{L}$ at the standard $1 / 10$ analyzer dilution (Figure 2). Assay imprecision was calculated

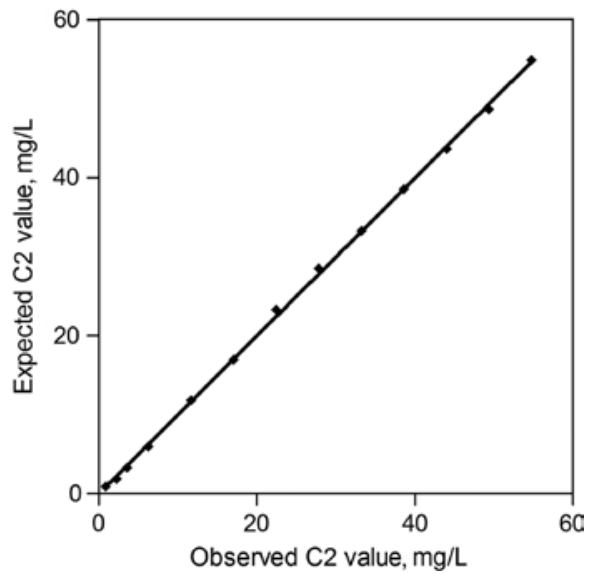

Figure 2: Linearity of the automated C2 assay. Thirteen samples were generated by diluting an EDTA-plasma sample pool with a high C2 concentration in an EDTA-plasma sample pool with a low concentration. Samples were run in triplicate and the mean result for each concentration was plotted against the expected $\mathrm{C} 2$ concentration. between runs, days, batches and instruments (Table 1). The within-run and between-run imprecisions for all five samples were less than $4 \%$. Between-batch and instrument imprecision were less than $7 \%$, between day $<10 \%$ and total precision $<11 \%$. The addition of chyle, hemoglobin, bilirubin, triglyceride or intralipid caused minimal interference with the measurement of $\mathrm{C} 2$ protein (Table 2).

\section{C2 protein reference range}

The median and mean concentrations in healthy adult blood donors ( $\mathrm{n}=120 ; 60$ males, 60 females) were 30.8 and $30.6 \mathrm{mg} / \mathrm{L}$, respectively, and the central 95th percentile reference interval was 18.9-42.7 mg/L. Skewness and kurtosis of the distribution were -0.1 and -0.8 , respectively. No significant difference was observed between sexes.

\section{Correlation between $\mathrm{C} 2$ concentrations measured in EDTA-plasma and serum}

C2 protein concentrations were measured in 119 matched EDTA-plasma and serum samples. There was a strong correlation between the observed concentrations in the two matrices $\left(\mathrm{R}^{2}=0.82, \mathrm{p}<0.0001, \mathrm{y}=1.03 \times-0.52 \mathrm{mg} / \mathrm{L}\right)$ with a mean negative bias in serum compared to plasma of $-0.3 \mathrm{mg} / \mathrm{L}$ ( $95 \%$ CI -8.4 to $7.8 \mathrm{mg} / \mathrm{L}$ ) (Figure 3). There was $98 \%$ agreement between the two measurements in classifying patients as below or above the lower limit of the normal range. Sample stability in the two matrices was assessed in 20 paired EDTA-plasma and serum samples from healthy donors (Supplementary Figure 1). Correlation between the two matrices was very strong where samples were assayed directly after collection $\left(\mathrm{R}^{2}=0.98\right.$,

Table 1: Imprecision of automated SPAPLUS ${ }^{\circledR}$ C2 assay.

\begin{tabular}{|c|c|c|c|c|c|c|c|c|c|c|c|c|}
\hline & \multicolumn{2}{|c|}{ Within-run } & \multicolumn{2}{|c|}{ Between-run } & \multicolumn{2}{|c|}{ Between-day } & \multicolumn{2}{|c|}{ Between-batch } & \multicolumn{2}{|c|}{$\begin{array}{l}\text { Between- } \\
\text { instrument }\end{array}$} & \multicolumn{2}{|c|}{ Total precision } \\
\hline & $\% \mathrm{CV}$ & SD & $\% \mathrm{CV}$ & SD & $\% \mathrm{CV}$ & SD & $\% \mathrm{CV}$ & SD & $\% \mathrm{CV}$ & SD & $\% \mathrm{CV}$ & SD \\
\hline Low & 3.2 & 0.25 & 2.4 & 0.18 & 9.3 & 0.70 & 0.7 & 0.16 & 3.8 & 0.89 & 10.9 & 0.82 \\
\hline Mid & 2.7 & 0.56 & 0.0 & 0.00 & 4.3 & 1.01 & 0.7 & 0.16 & 5.9 & 1.44 & 5.5 & 1.31 \\
\hline High & 2.7 & 0.92 & 1.6 & 0.56 & 7.4 & 2.56 & 6.2 & 2.12 & 5.3 & 1.80 & 7.8 & 2.67 \\
\hline$-25 \%$ & 3.8 & 0.29 & 3.2 & 0.29 & 6.9 & 0.64 & 3.6 & 0.33 & 4.9 & 0.45 & 8.0 & 0.74 \\
\hline$+25 \%$ & 2.3 & 0.27 & 3.3 & 0.37 & 5.3 & 0.59 & 2.3 & 0.25 & 5.0 & 0.56 & 6.7 & 0.75 \\
\hline
\end{tabular}

Precision was assessed in EDTA-plasma samples with five different C2 concentrations (low, $7.7 \mathrm{mg} / \mathrm{L}$; mid, $21.2 \mathrm{mg} / \mathrm{L}$; high, $35.2 \mathrm{mg} / \mathrm{L}$; $25 \%$ medical decision point, $7.9 \mathrm{mg} / \mathrm{L}$; and $+25 \%$ medical decision point, $11.5 \mathrm{mg} / \mathrm{L}$ ). $\% \mathrm{CV}$ and SD for within-run precision were calculated from 20 measurements per sample. For the other precision calculations, each sample was assayed in duplicate, with two runs per day over 21 days, giving a total of 84 readings per sample. This part of the study was carried out using three reagent lots and three different analyzers. 
Table 2: Interference data for C2 automated assay.

\begin{tabular}{lrrrrr}
\hline & Chyle & Bilirubin & Hemoglobin & Triglyceride & Intralipid \\
\hline Low & -0.57 & -2.51 & 1.43 & 1.38 & 8.03 \\
MDP & -0.92 & -3.33 & 2.42 & 7.90 & -1.28 \\
Normal range & 1.15 & -5.46 & -0.32 & -0.16 & 5.15 \\
\hline
\end{tabular}

ETDA-plasma samples with three different $\mathrm{C} 2$ concentrations were spiked with one of the five interferents or saline as a control. Samples tested include one low concentration sample, one around the medical decision point (MDP) and one within the normal range (for hemoglobin, bilirubin and chyle: 7.8, 10.4 and $27.8 \mathrm{mg} / \mathrm{L}$; for intralipid and triglyceride; 7.5, 10.5 and $25.1 \mathrm{mg} / \mathrm{L}$ ). All samples were then run in the SPAPLUS ${ }^{\circledR} \mathrm{C} 2$ assay. Values represent percentage change observed in the presence of the interfering substances.

Passing and Bablok fit $\mathrm{y}=1.0 \times+1.0)$, and slightly weaker where samples had been stored for 3 days at $4{ }^{\circ} \mathrm{C}\left(\mathrm{R}^{2}=0.89\right.$, Passing and Bablok fit $\mathrm{y}=1.2 \times-1.9$ ).

\section{Correlation between $\mathrm{C} 2$ concentrations measured using the automated turbidimetric and RID assays}

The $\mathrm{C} 2$ concentrations were measured in 24 healthy controls and seven patients with confirmed or suspected C2 deficiencies by both the $\mathrm{C} 2$ automated turbidimetric and RID assays. There was a correlation between the assays $\left(\mathrm{R}^{2}=0.89, \mathrm{p}<0.0001, \mathrm{y}=1.07 \times+2.26\right)$, with the automated assay having a mean positive bias of $4.5 \mathrm{mg} / \mathrm{L}$ (95\% CI -2.5 to $11.5 \mathrm{mg} / \mathrm{L}$ ) (Figure 4). There was $94 \%$ agreement between the two assays as classifying individuals above or below the lower limit of the respective measuring ranges.

\section{Correlation between $\mathrm{C} 2$ concentrations and functional complement activity}

The C2 concentration in the 119 EDTA-plasma samples was compared with $\mathrm{CH} 50$ activity in the matched serum samples (Figure 5). There was a significant correlation between $\mathrm{C} 2$ protein concentration and $\mathrm{CH} 50$ activity, with $98.3 \%$ agreement in classifying patients as below or above the lower limit of the assays normal ranges $(\mathrm{p}<0.0001$, $\left.\mathrm{R}^{2}=0.48, \mathrm{y}=0.6 \times-7\right)$

\section{Clinical study - C2 concentrations in various clinical samples}

The $\mathrm{C} 2$ concentration was quantified in samples from an independent group of normal healthy controls, patients with homozygous or heterozygous C2 deficiency, AAE, SLE and other clinical samples from patients with chronic, inflammatory conditions such as coeliac disease and Sjögren's syndrome. The independent group of normal healthy controls validated the adult normal reference range. Significant differences were observed between the median C2 concentrations obtained in the normal controls and both the patients with homozygous $\mathrm{C} 2$ deficiency $(p<0.01)$ and the inflammatory patient group $(p<0.0001)$ (Figure 6). Both AAE patients had C2 concentrations below the normal reference range $(18.8 \mathrm{mg} / \mathrm{L}$ and $6.8 \mathrm{mg} / \mathrm{L}$ ), whereas the heterozygous C2-deficient individual had a $\mathrm{C} 2$ concentration within, but at the lower end, of the normal reference range $(25.5 \mathrm{mg} / \mathrm{L})$. In the SLE cohort, nine patients had normal concentrations of $\mathrm{C} 2$, two patients had concentrations below $(9.2$ and $6.6 \mathrm{mg} / \mathrm{L})$
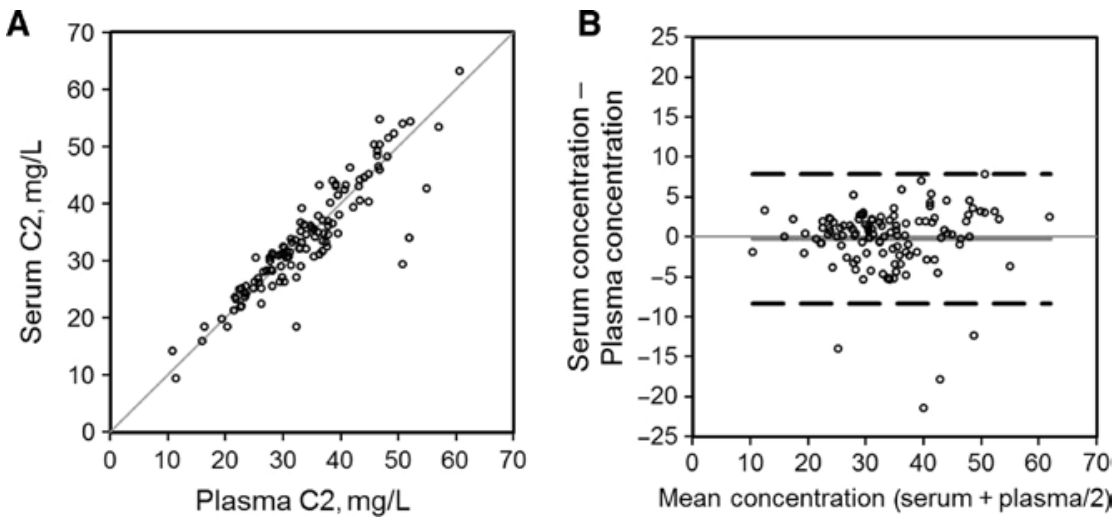

Figure 3: Comparison of the $\mathrm{C} 2$ concentration measured in EDTA-plasma and serum samples. The C2 concentration was measured in 119 matched serum and EDTA-plasma samples. The comparison between the two matrices was assessed using (A) Passing-Bablok analysis (identity line shown where $y=x$ ) and (B) Bland-Altman analysis (solid line demonstrates the mean difference and broken lines show the limits of agreement, -1.96 and +1.96 SDs). 

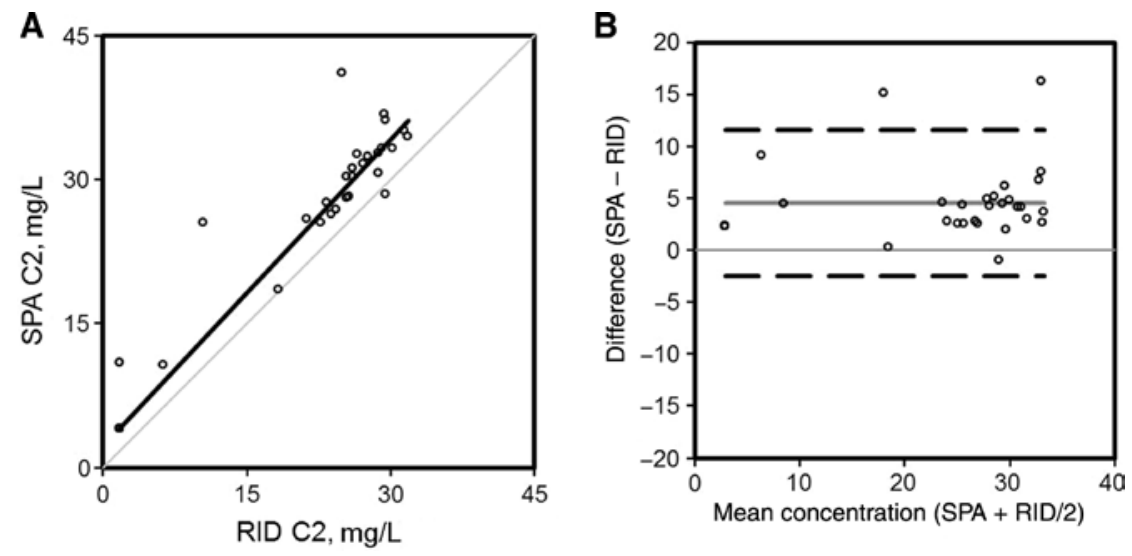

Figure 4: Comparison of the C2 concentration measured by the C2 SPAPLUS ${ }^{\circledR}$ assay and RID.

The C2 concentration was measured by both the C2 SPAPLUS ${ }^{\circledR}$ assay and RID in 24 healthy controls and seven individuals with confirmed or suspected C2 deficiency. The comparison between the two methods was assessed using (A) Passing-Bablok analysis (identity line shown where $y=x$ ) and (B) Bland-Altman analysis (solid line demonstrates the mean difference and broken lines show the limits of agreement, -1.96 and +1.96 SDs).

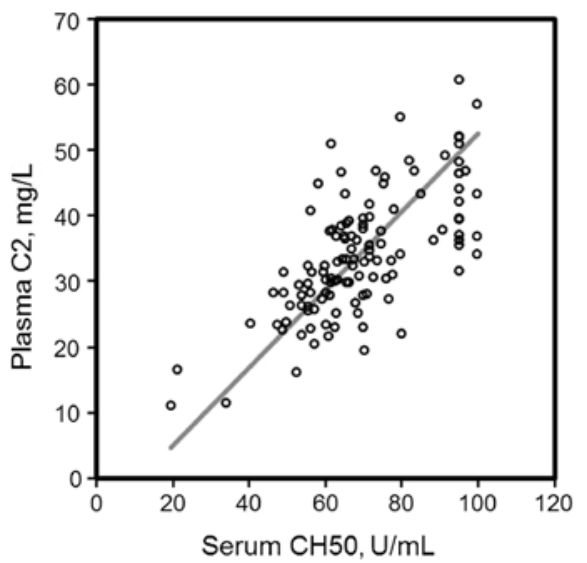

Figure 5: Correlation between C2 concentration and functional CH50 activity.

The $\mathrm{C} 2$ concentration (EDTA-Plasma) and $\mathrm{CH} 50$ activities (serum) were quantified in 119 matched patient samples. Results were analyzed using Passing-Bablok analysis.

and two patients had concentrations above $(52.8$ and $69.6 \mathrm{mg} / \mathrm{L}$ ) the normal reference range.

\section{Discussion}

In the present study, we have developed an automated C2 assay for use on the SPAPLUS ${ }^{\circledR}$ turbidimetric analyzer. We produced C2-specific antisera using purified intact C2 antigen as it is known that the fragments of the protein can be structurally diverse from the parent molecule. The method used here has enabled the rapid, precise and quantitative determination of $\mathrm{C} 2$ protein concentration over a

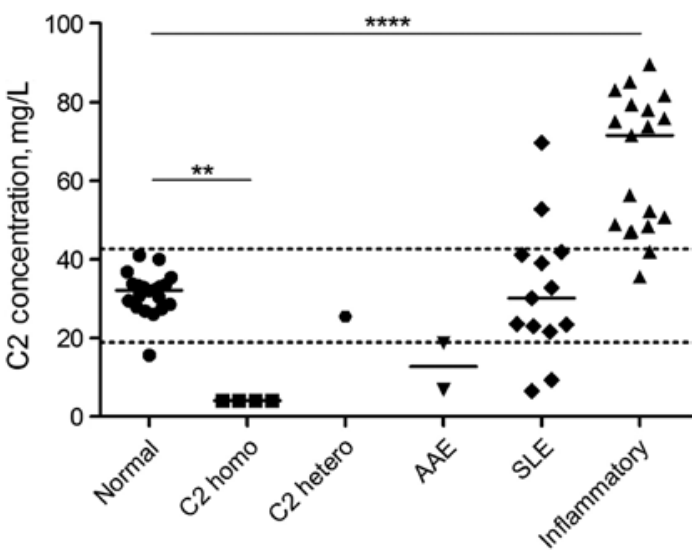

Figure 6: $\mathrm{C} 2$ concentrations in clinical samples. The $\mathrm{C} 2$ concentration was quantified in serum samples from normal, healthy controls $(n=20)$, homozygous C2-deficient patients ( $C 2$ homo, $n=4$ ), a heterozygous $C 2$-deficient individual ( 22 hetero, $n=1$ ), acquired angioedema patients ( $A A E, n=2)$, systemic lupus erythematosus patients (SLE, $\mathrm{n}=13$ ) and other clinical samples from a referral population with chronic, inflammatory conditions $(n=19)$. Dotted lines demonstrate the upper and lower limits of the normal range. ${ }^{\star \star} p<0.01,{ }^{\star \star \star \star} p<0.0001$.

wide measuring range, a range likely to be encountered in clinical practice. The automated C2 assay was linear over the range $2.3-54.8 \mathrm{mg} / \mathrm{L}$ at the standard sample dilution and was unaffected by common blood constituents which might interfere in the assay.

A major consideration when measuring complement proteins and their activation products is that complement activation can occur ex vivo. EDTA inhibits complement activation, so EDTA-plasma is often considered a more stable matrix than serum for complement evaluations 
$[22,23]$. The comparison of $\mathrm{C} 2$ protein concentrations measured in EDTA-plasma and serum samples here demonstrates a high level of agreement between the two matrices for this assay. There are four samples outside the Bland-Altman established criteria. These samples could not be assayed on the day of collection, so this may be due to the stability of C2 measurements in serum. Indeed, in the assessment of stability in the two matrices performed here, the correlation did weaken slightly after storage of samples. The manufacturer's recommendation is that serum may be used to measure $\mathrm{C} 2$ concentrations in this assay where samples will be processed and tested on the day of collection, but where this cannot be guaranteed, EDTA-plasma is the more suitable matrix.

A number of laboratories are using techniques such as electroimmunoassay to measure C2 concentrations [19]. This technique can be laborious and time consuming, and the interpretation of results is potentially subjective, requiring trained staff to carry out the analyses. The assay we have described here is automated, simple and has a quick time to first result. The assay has also fulfilled criteria for linearity, precision and interference, which are based on those suggested by the Clinical Laboratory and Standards Institute, making it an attractive alternative to other available methods. The automated C2 assay shows a significant correlation with $\mathrm{CH} 50$ activity, and with $\mathrm{C} 2$ concentrations measured by the traditional RID method.

Using healthy adult blood donors, we developed a 95th percentile reference interval of $18.9-42.7 \mathrm{mg} / \mathrm{L}$ and validated it with an independent group of healthy samples. All four patients with previously determined genetic C2 deficiency had a C2 concentration of $<3.8 \mathrm{mg} / \mathrm{L}$. Patient 1 and patient 2 had the most common $\mathrm{C} 2$ deficiency, with a confirmed homozygous 28-bp deletion. Both of these patients, as would be expected, had abnormal $\mathrm{CH} 50$ results but normal AP50 activities. The individual with a heterozygous C2 deficiency had $25.5 \mathrm{mg} / \mathrm{L} \mathrm{C2}$, which is within but at the lower end of the normal range. Testing a larger cohort of heterozygous patients on this assay is necessary to establish a potential range of $\mathrm{C} 2$ concentrations in heterozygous-deficient individuals.

Measuring C2 concentrations is important in the evaluation of patients with a reduced $\mathrm{CH} 50$ test result, and in the assessment of potential homozygous and heterozygous $\mathrm{C} 2$ deficiency. The measurement of C2 may also have a utility in patients with SLE and is reduced in hereditary and acquired angioedema. Here we have shown that the two AAE patients tested had $\mathrm{C} 2$ concentrations below the normal reference range. These individuals were also known to have low C4 concentrations $(0.02 \mathrm{~g} / \mathrm{L}$ and $<0.01$ $\mathrm{g} / \mathrm{L}$, respectively). Donaldson and Rosen [24] demonstrated that $\mathrm{C} 2$ and $\mathrm{C} 4$, but not $\mathrm{C} 3$, were completely diminished in patients with hereditary angioedema (HAE) during an attack. Following recovery these levels increased, but still remained low. Patients with HAE or AAE related to $\mathrm{C} 1$ inhibitor have absent or reduced levels of $\mathrm{C} 1$ inhibitor, or have protein which does not function normally. This leads to uncontrolled activation of the complement and contact systems. Patients suffer from uncontrolled, sometimes life-threatening, episodes of edema, which can be treated if correctly diagnosed, and prophylactic medications prescribed if attacks are severe and recurrent. Low levels of C2 and C4 with normal C3 concentrations are highly characteristic of HAE [25], with C4 measurements often used as a front line test, as it is simple test and readily available. More recently, it has been shown that C4 can be normal in a small proportion of HAE patients (including those on therapy) [26, 27], so it would be interesting to study C2 in these individuals.

It has also been shown that $\mathrm{C} 2$ expression can be abnormal in autoimmune disease. Here, 2/13 serum samples from SLE patients had C2 concentrations $<50 \%$ below the lower limit of the reference range and 2/13 had concentrations above the upper limit of the reference range. Soter et al. [28] demonstrated that the early classical pathway components (C1, C4 and C2) were often depressed in sera from individuals with rheumatoid arthritis (RA), Sjögren's syndrome or SLE. On the other hand, Firestein et al. [29] showed that C2 messenger RNA was increased in the synovium of patients with RA, and this correlated with the degree of synovial inflammation. In our study, 31\% of SLE patients had serum C2 concentrations outside of the normal reference range, with patients exhibiting both reduced and elevated levels. Complement proteins are acute phase proteins, and their expression is increased in certain inflammatory conditions. Furthermore, homozygous and heterozygous C2 deficiencies are associated with increased incidence of SLE [30]. Our data suggest that in some SLE patients tested, there is an acute phase response, as indicated by the elevated C2 levels. A simple automated assay to measure C2 may aid in the evaluation of these patients.

One of the key limitations of this study is that the normal range could not be assessed in pediatric samples. Immunodeficiency is often tested in children less than 3 years of age, so future studies developing the normal reference intervals for $\mathrm{C} 2$ in this age-group would be useful. There are, however, previous studies looking at C2 levels in infants using RID techniques. Davis et al. [31] demonstrated in 10 infants at 1 month old and 10 infants aged 6 months that the $\mathrm{C} 2$ concentrations were comparable to that found in adults. 
In conclusion, we have developed a simple, automated assay that can measure $\mathrm{C} 2$ protein concentration in plasma and serum samples. $\mathrm{C} 2$ concentrations in disease have not yet been as extensively studied as other complement proteins, such as $\mathrm{C} 3$ and $\mathrm{C} 4$, but the development of this simple, rapid and reliable method will provide the platform for such future studies.

Author contributions: All the authors have accepted responsibility for the entire content of this submitted manuscript and approved submission.

Research funding: None declared.

Employment or leadership: CET, BJB, AC, SH, GW and ARP are employees of The Binding Site.

Honorarium: None declared.

Competing interests: The funding organization(s) played no role in the study design; in the collection, analysis, and interpretation of data; in the writing of the report; or in the decision to submit the report for publication.

\section{References}

1. Morley BJ, Walport M. The complement factsbook. San Diego, CA: Academic Press, 2000:viii, 228.

2. Volanakis JE, Frank MM. The human complement system in health and disease. New York: M. Dekker, 1998:ix, 656.

3. Tegla CA, Cudrici C, Patel S, Trippe R 3rd, Rus V, Niculescu F, et al. Membrane attack by complement: the assembly and biology of terminal complement complexes. Immunol Res 2011;51:45-60.

4. Song WC, Sarrias MR, Lambris JD. Complement and innate immunity. Immunopharmacology 2000;49:187-98.

5. Dunkelberger JR, Song WC. Complement and its role in innate and adaptive immune responses. Cell Res 2010;20:34-50.

6. Polley MJ, Muller-Eberhard HJ. The second component of human complement: its isolation, fragmentation by C'1 esterase, and incorporation into C'3 convertase. J Exp Med 1968;128:533-51.

7. Vorup-Jensen T, Petersen SV, Hansen AG, Poulsen K, Schwaeble W, Sim RB, et al. Distinct pathways of mannan-binding lectin (MBL)- and C1-complex autoactivation revealed by reconstitution of MBL with recombinant MBL-associated serine protease-2. J Immunol 2000;165:2093-100.

8. Jonsson H, Sturfelt G, Martensson U, Truedsson L, Sjoholm AG. Prospective analysis of $\mathrm{C} 1$ dissociation and complement activation in patients with systemic lupus erythematosus. Clin Exp Rheumatol 1995;13:573-80.

9. Swaak AJ, Aarden LA, Statius van Eps LW, Feltkamp TE. AntidsDNA and complement profiles as prognostic guides in systemic lupus erythematosus. Arthritis Rheum 1979;22:226-35.

10. Jonsson G, Truedsson L, Sturfelt G, Oxelius VA, Braconier JH, Sjoholm AG. Hereditary C2 deficiency in Sweden: frequent occurrence of invasive infection, atherosclerosis, and rheumatic disease. Medicine (Baltimore) 2005;84:23-34.
11. Ross SC, Densen P. Complement deficiency states and infection: epidemiology, pathogenesis and consequences of neisserial and other infections in an immune deficiency. Medicine (Baltimore) 1984;63:243-73.

12. Klemperer MR, Woodworth HC, Rosen FS, Austen KF. Hereditary deficiency of the second component of complement (C'2) in man. J Clin Invest 1966;45:880-90.

13. Johnson CA, Densen P, Hurford RK, Jr., Colten HR, Wetsel RA. Type I human complement $C 2$ deficiency. A 28-base pair gene deletion causes skipping of exon 6 during RNA splicing. J Biol Chem 1992;267:9347-53.

14. Agnello V, De Bracco MM, Kunkel HG. Hereditary C2 deficiency with some manifestations of systemic lupus erythematosus. J Immunol 1972;108:837-40.

15. Pickering MC, Walport MJ. Links between complement abnormalities and systemic lupus erythematosus. Rheumatology (Oxford) 2000;39:133-41.

16. Densen P. Complement deficiencies and meningococcal disease. Clin Exp Immunol 1991;86(Suppl 1):57-62.

17. De Witt CC, Ascher DP, Winkelstein J. Group B streptococcal disease in a child beyond early infancy with a deficiency of the second component of complement (C2). Pediatr Infect Dis J 1999;18:77-8.

18. Skattum L, van Deuren M, van der Poll T, Truedsson L. Complement deficiency states and associated infections. Mol Immunol 2011;48:1643-55.

19. Johnson U, Truedsson L, Gustavii B. Complement components in 100 newborns and their mothers determined by electroimmunoassay. Acta Pathol Microbiol Immunol Scand C 1983;91:147-50.

20. Sonntag J, Brandenburg U, Polzehl D, Strauss E, Vogel M, Dudenhausen JW, et al. Complement system in healthy term newborns: reference values in umbilical cord blood. Pediatr Dev Pathol 1998;1:131-5.

21. Schlesinger M, Broman I, Lugassy G. The complement system is defective in chronic lymphatic leukemia patients and in their healthy relatives. Leukemia 1996;10:1509-13.

22. Kirschfink M, Mollnes TE. Modern complement analysis. Clin Diagn Lab Immunol 2003;10:982-9.

23. Mollnes TE, Jokiranta TS, Truedsson L, Nilsson B, Rodriguez de CS, Kirschfink M. Complement analysis in the 21st century. Mol Immunol 2007;44:3838-49.

24. Donaldson VH, Rosen FS. Action of complement in hereditary angioneurotic edema: the role of C'1-esterase. J Clin Invest 1964;43:2204-13.

25. Davis AE 3rd. The pathophysiology of hereditary angioedema. Clin Immunol 2005;114:3-9.

26. Tarzi MD, Hickey A, Forster T, Mohammadi M, Longhurst HJ. An evaluation of tests used for the diagnosis and monitoring of $C_{1}$ inhibitor deficiency: normal serum C4 does not exclude hereditary angio-oedema. Clin Exp Immunol 2007;149:513-6.

27. Aabom A, Bygum A, Koch C. Complement factor C4 activation in patients with hereditary angioedema. Clin Biochem 2017;50:816-21.

28. Soter NA, Austen KF, Gigli I. The complement system in necrotizing angiitis of the skin. Analysis of complement component activities in serum of patients with concomitant collagen-vascular diseases. J Invest Dermatol 1974;63:219-26.

29. Firestein GS, Paine MM, Littman BH. Gene expression (collagenase, tissue inhibitor of metalloproteinases, complement, 
and HLA-DR) in rheumatoid arthritis and osteoarthritis synovium. Quantitative analysis and effect of intraarticular corticosteroids. Arthritis Rheum 1991;34:1094-105.

30. Macedo AC, Isaac L. Systemic lupus erythematosus and deficiencies of early components of the complement classical pathway. Front Immunol 2016;7:55.
31. Davis CA, Vallota EH, Forristal J. Serum complement levels in infancy: age related changes. Pediatr Res 1979;13:1043-6.

Supplementary Material: The online version of this article offers supplementary material (https://doi.org/10.1515/cclm-2017-1068). 